\title{
(+)-生物素全合成研究新进展
}

\author{
钟 铮 $*, a$ 武雪芬 ${ }^{a}$ 陈芬儿 $*, b$ \\ ( ${ }^{a}$ 河南中医学院药学院 郑州 450008) \\ ( $b$ 复旦大学化学系 上海 200433)
}

\begin{abstract}
摘要 (+)-生物素是维生素 $\mathrm{B}$ 家族中的一员, 自发现以来对其全合成的报道层出不穷. 在最近十几年中, 数十条新的 合成路线和改进方法陆续报道. (+)-生物素全合成策略主要分为两类: 对映选择性合成和立体专一性合成. 前一策略 中, 通过各种反应方法对经典的 Hoffmann-La-Roche-硫内酯法进行改进和完善，其中不对称催化合成的方法已成功应 用于工业化生产; 在后一策略中, 以 $L$-半胱氨酸为起始原料的合成途径得到了较大发展, 正越来越具有工业意义. 关键词 (十)-生物素; 全合成; 研究新进展
\end{abstract}

\section{Recent Progresses in Total Synthesis of $(+)$-Biotin}

\author{
Zhong, Zheng ${ }^{*, a} \quad \mathrm{Wu}$, Xuefen $^{a} \quad$ Chen, Fener ${ }^{*, b}$ \\ ( ${ }^{a}$ School of Pharmcy, Henan University of Traditional Chinese Medicine, Zhengzhou 450008) \\ ( ${ }^{b}$ Department of Chemistry, Fudan University, Shanghai 200433)
}

\begin{abstract}
Biotin is one of the B vitamins. The study on total synthesis of $(+)$-biotin is an incessant pursuit since it was discovered half century ago. During the past ten years great advances in this field have been made. The synthetic approaches toward the target molecule can be classified into two series: the enatioselective syntheses and the stereospecific syntheses. In former approach, modification of the already existed Hoffmann-La-Roche's lactone-thiolactone approach was further developed in diversified ways, especially the great advance in asymmetric synthesis of the chiral framework of $(+)$-biotin, which had been put into industrial practice successfully; in terms of the latter approach, $L$-cysteine or cystine can be regarded as more logical starting materials. Enriched by a plenty of ingenious novel strategies and tactics, this approach evolved more facile and practical than before.
\end{abstract}

Keywords (+)-biotin; total synthesis; progress

$(+)$-生物素是维生素 B 家族中的一员，也被称为维 生素 $\mathrm{H}$ 或辅酶 $\mathrm{R}^{[1]}$. 在自然界中, $(+)$-生物素广泛分布于 动植物体内. 它在脂肪酸生化合成、糖酵解等多种生化 反应中扮演重要角色, $(+)$-生物素的缺乏会引起生长缓 慢等营养性疾病, 因此 $(+)$-生物素是重要的医药产品和 饲料添加剂 ${ }^{[2]}$. 此外它还是分子生物学中常用的分子探 针标记物. 由于医药市场对 $(+)$-生物素的巨大需求, 使 得 $(+)$-生物素的全合成也成为了众多合成化学家的不 解追求 (Scheme 1).

1997 年比利时教授 Clercq ${ }^{[3]}$ 曾经对 $(+)$-生物素近半 个世纪的全合成研究做过精彩的评述, 在过去的十几年 中, 企业界和学术界对 $(+)$-生物素兴趣丝毫没有减弱,

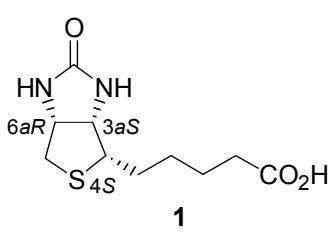

\section{Scheme 1}

随着新合成技术和方法的不断涌现，化学家对 $(+)$-生物 素全合成的思路不断拓展, 将 $(+)$-生物素的合成研究也 推向了一个全新的高度. 本文对最近十几年中在 $(+)$-生 物素全合成领域内的研究进展做一综述.

到目前为止所有 $(+)$-生物素的全合成策略可基本 分为两种：对映选择性合成和立体专一性合成. 前者是

\footnotetext{
*E-mail: zhongzski@yahoo.cn

Received March 4, 2012; revised May 14, 2012; published online May 22, 2012.

Project supported by the Doctoral Scientific Foundation of Henan University of Traditional Chinese Medicine (No. BSJJ2010-28). 河南中医学院 “博士科研基金” (No. BSJJ2010-28)资助项目.
} 
采用富马酸为原料, 通过不对称合成或其他手性技术构 建 $(3 a S, 4 S, 6 a R)$ 三个手性碳, 经多步反应最终获得足够 光学纯度的产品. 另一策略是采用 $L$-半胱氨酸等手性化 合物作为起始原料, 利用原料中的手性碳原子, 通过结 构变换得到 $(+)$-生物素. 由于起始原料具有手性, 所以 也称为手性源策略. 本文将从这两个方面展开讨论.

\section{1 对映选择性合成策略}

早在 20 世纪 40 年代 Hoffmann-La-Roche 公司的化 学家 Sternbach 和 Goldberg 首次报道了(+)-生物素的全 合成. 正是他们的开创性工作奠定了之后的 $(+)$-生物素 工业化合成路线的基础, 该路线因此也被称为 Sternbach 路线或 Hoffmann-La-Roche (HLR) 路线 ${ }^{[4]}$. 之 后的几十年里, 无论企业界还是学术界的化学家都想方 设法通过改进这条路线降低(+)-生物素的生产成本.

2001 年 Chen 小组 ${ }^{[5]}$ 对 HLR 内酯-硫内酯路线进行 改进, 报道了一条较为完整的工业化合成路线(Scheme 2).

在该路线中, 环酸䣶 $\mathbf{3}$ 通过内消旋二酸 $\mathbf{2}$ 脱水得到, 3 酯化后得到外消旋的半酯 4. 用( $S, S$ )-2-氨基-1-(4-硝基 苯基)-1,3-丙二醇(生产氯霉素中间体时产生的副产物) 作拆分剂, 通过外消旋半酯 $\mathbf{4}$ 与拆分剂形成的非对映异 构体盐重结晶得到所需构型的半酯 $\mathbf{4}$. 半酯 $\mathbf{4}$ 经原位生 成的嗍氢化锂还原后环合, 得到 $(3 a S, 6 a R)$-内酯 $\mathbf{5}$. 内酯 5 可以被硫代乙酸钾在乙酸钾存在下硫代为关键中间体 (3aS,6aR)-硫内酯 6. 羧基丁基侧链是通过格氏反应引入 的. 甲氧基丙基澳格氏试剂与硫内酯 $\mathbf{6}$ 偶联后再经酸性 脱水得到环外烯烃 7. 采用可方便回收的树脂固载钯为 催化剂, 甲酸铵作质子源, 通过催化转移氢化将环外烯 烃 7 还原为醚 8 , 由于环外烯烃 $\mathbf{7}$ 中氮原子上芐基保护 基位阻的原因, 氢化反应立体选择性的得到了 (3aS, $4 S, 6 a R)$ 构型的产物. 为了将醚 $\mathbf{8}$ 上甲基脱去, 使用 了 $35 \%$ 的氢溴酸进行回流, 同时加入催化量的新洁尔 灭, 硫原子较好的亲核性使得醚 8 顺利转化溴化硫鎓盐 9(采用氯化氢与甲酸的混合液进行回流得到相应的氯 化硫鎓盐 ${ }^{[6]}$ ). 在弱碱碳酸钾的作用下, 由丙二酸二乙酯 的形成的碳负离子与硫鎓盐 9 发生亲核取代反应得到二 酯 10 , 后者在澳化氢一乙酸中很容易的发生水解脱羧反 应，同时氮原子上的两个苄基保护基也一并脱去，“一 锅反应” 得到目标产物 $(+)$-生物素, 总收率 14.5\%。

该路线代表性的涵盖了 HLR 路线各种改进形式的 基本特征, 即整个路线可以分为三个主要阶段：(1) $(3 a S, 6 a R)$-内酯 $\mathbf{5}$ 或( $3 a S, 6 a R)$-硫内酯 $\mathbf{6}$ 的获得; (2)羒基 丁基侧链的引入; (3)芐基保护基的高产率脱去. 几乎所 有对 HLR 路线的改进研究都是针对这三个阶段开展
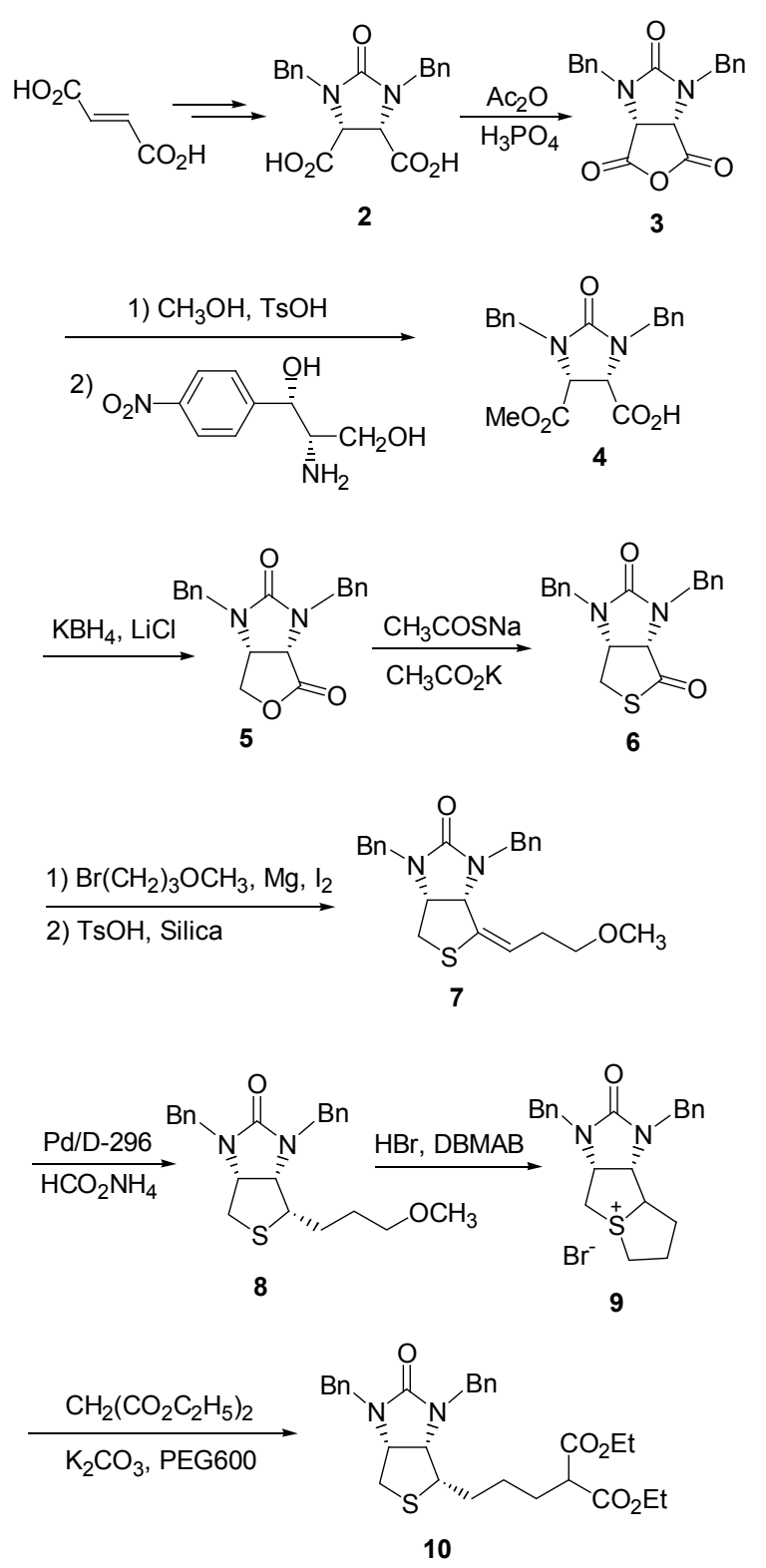

$\mathrm{HBr}, \mathrm{CH}_{3} \mathrm{CO}_{2} \mathrm{H}$ 1

\section{Scheme 2}

的, 因此对 HLR 路线的讨论也由这三个主题展开.

\section{$1.1(3 a S, 6 a R)$-内酯 5 或 $(3 a S, 6 a R)$-硫内酯 6 的立体选 择性获得}

高产率高 $e e$ 值的得到内酯 5 或硫内酯 $\mathbf{6}$ 是 HLR 路 线中最具有挑战性的工作. 在最近十几年中发展出各具 特色的不对称合成方法.

1993 年 Matsuki 等 ${ }^{[7]}$ 曾使用 Noyori 小组发展出的铝 锂氢-联菜酚试剂(BINAL-H)成功将内消旋二酸䣲 $\mathbf{3}$ 高 $e e$ 值的还原为内酯 5. 2002 年 Chen 小组 ${ }^{[8]}$ 也同样用 $(R)$-BINAL-H 还原了内消旋硫代酸䣶得到中间体硫内 酯 6, 收率 $83 \%$, ee 值达 $98.5 \%$ (Scheme 3). 


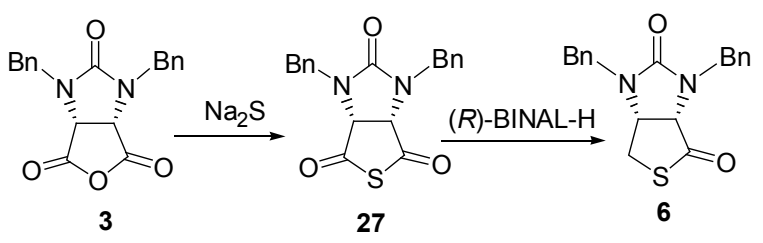

Scheme 3

使用手性试剂的方法虽然能够得到理想的产率和 $e e$ 值, 但并不经济因而没有工业应用前景.

1997 年有文献报道了使用苏氨酸衍生物噁唑硼烷 与硼烷-THF 的混合物可以对映选择性的还原内消旋酰 亚胺为羟基内酰胺的方法 ${ }^{[9]}$. 很快 1999 年 Shimizu 等 ${ }^{[10]}$ 就成功将该方法用于(+)-生物素前体, (+)-脱氧生物素 的不对称合成中. 该方法首先将内消旋的二酸 2 转化为 酰亚胺 11, 使用 0.5 equiv. 的噁唑硼烷和过量的硼烷作 还原剂以 $98 \%$ 的 $e e$ 值和 $65 \%$ 的收率得到羟基内酰胺 $\mathbf{1 2}$, 在用硼氢化钠还原和酸性水解之后环合得到内酯 $\mathbf{5}$ (Scheme 4).

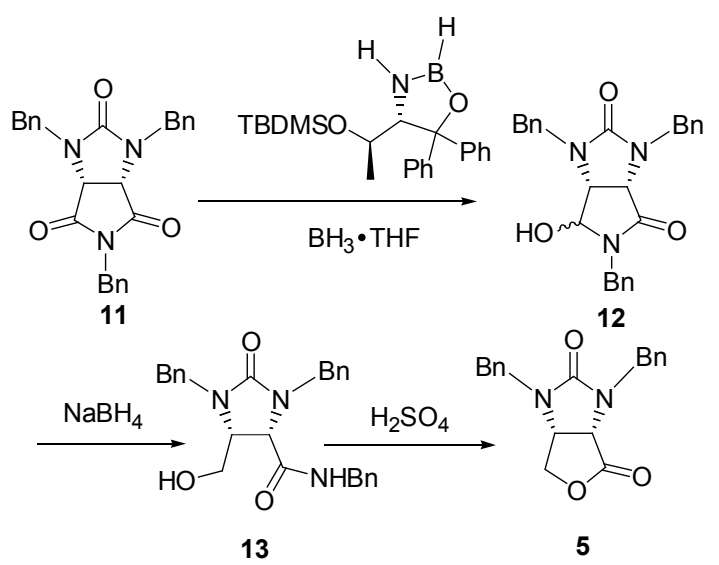

Scheme 4

由于硼烷复合物价格较高且储存和操作不便, 不适 于大规模制备使用. 2003 年在 Chen 小组 ${ }^{[1]}$ 使用氢化锂 与三氟化嗍 - 乙醚原位生成硼烷, 再加入配体 (1S,2S)-(+)-苏式-1-(4-硝基苯基)-2-氨基-1,3-丙二醇与 嗍烷原位生成噁唑嗍烷催化剂, 同样高立体选择性的将 酰亚胺 11 还原成着基内酰胺 12, 反应更加经济安全 (Eq. 1).

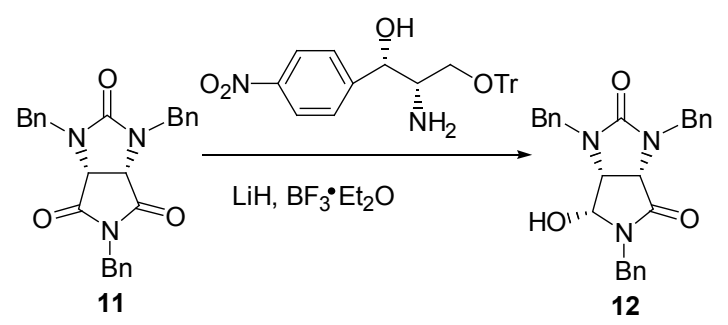

原位生成的噁唑硼烷催化剂采用手性氨基醇作为 配体，在大规模制备中这些水溶性很强的小分子配体回 收非常困难. Chen 小组 ${ }^{[12]}$ 后报道了采用树脂固载手 性配体制备的噁唑硼烷催化剂. $L$-脯氨醇手性配体与树 脂通过磺酰胺键相连，在过量的厥烷-THF 复合物体系 中原位生成噁唑硼烷, 将酰亚胺 11 还原为羟基内酰胺 12, 12 转化为内酯 5 后的 $e e$ 值可达 $98.5 \%$ (Eq. 2).

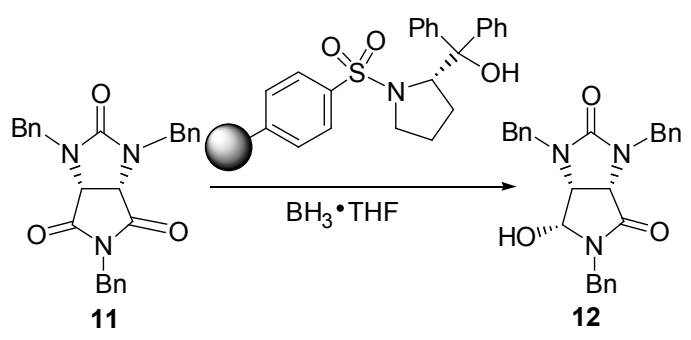

很快 Chen 小组 ${ }^{[13]}$ 又报道了使用 $\mathrm{NaH}$ 与三氟化硣乙醚原位生成硼烷，树脂固载手性氨基醇配体催化还原 酰亚胺 11 的方法, 使得该方法更加适用于大规模制备 (Eq. 3).

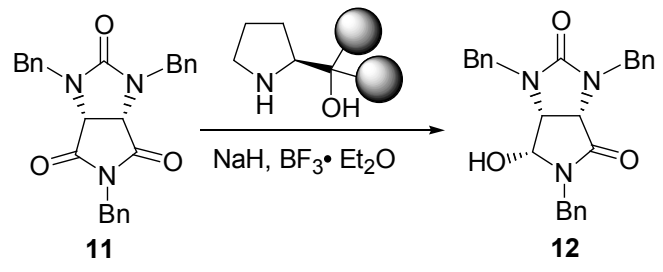

虽然惡唑硼烷催化还原获得内酯 $\mathbf{5}$ 的方法取得了很 大成功，但是无水无氧的反应条件对工业化生产来说还 是较为苛刻的.

为了更适于工业化生产，帝斯曼公司的研究人员 发展了一种通过手性助剂不对称酯化来获得内酯 5 的方 法. 酸䣶 3 与 $(S)$-1,1-二苯基-1,2-丙二醇酯化得到单酯 16, 单酯 16 被硼氢化锂还原后酸化环合得到内酯 5 , 收 率 $89 \%$, ee 值 $98.5 \%{ }^{[14]}$ (Scheme 5).
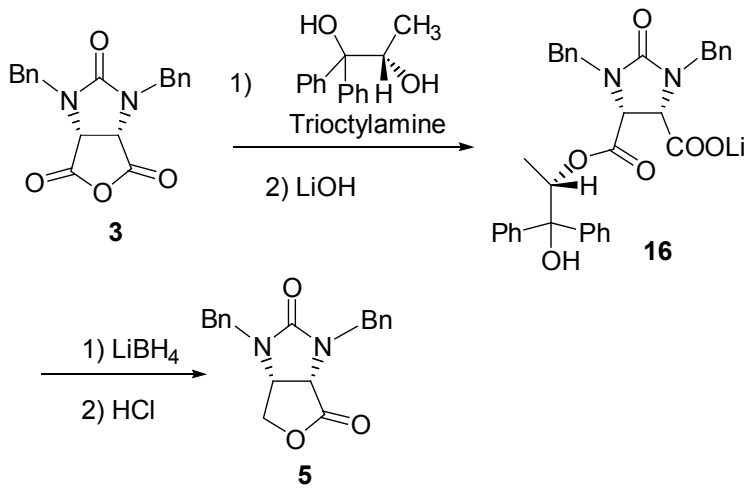

$$
\text { Scheme } 5
$$

默克公司的研究人员开发了另外一种不对称酯化 
的方法. 他们采用 $(1 R, 2 S)-(-)$-2-二甲基氨基-1-苯基-1丙醇等手性醇作为手性助剂, 与酸䣶 3 酯化后生成手性 单酯, 然后经过还原和酸化环合得到内酯 $\mathbf{5}$, ee 值为 $50 \% \sim 90 \%$ 不等 ${ }^{[15]}$ (Eq. 4).

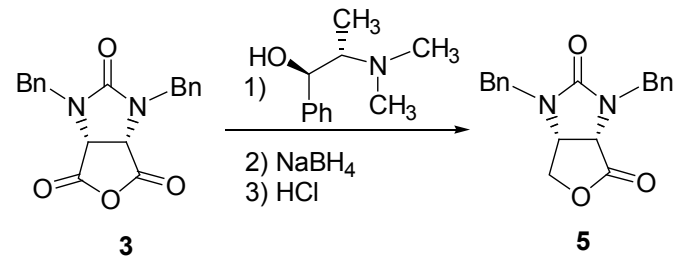

Deng 小组 ${ }^{[16]}$ 报道了一种利用金鸡纳碱衍生物催化 不对称合成内酯 $\mathbf{5}$ 的方法. 反应在二氢奎尼丁基菲 (DHQD-PHN) 催化下将环酸䣶 3 去对称化酯化得到半酯 4 的对映体 ent-4, 然后再采用改良 Falorini “一锅 法” ${ }^{[17]}$, 使用三聚氯氰、 $N$-甲基吗啉和硼氢化钠将 ent-4 还原后环合得到内酯 $\mathbf{5}, e e$ 值为 $91 \%$, 收率 $82 \%$ (Scheme 6 ).
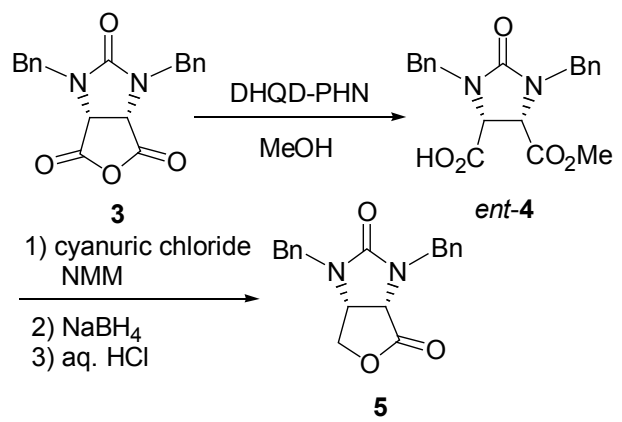

Scheme 6

为了获得更高的 $e e$ 值, Chen 小组 ${ }^{[18]}$ 对这一方法进 行了改进. 对金鸡纳碱催化剂结构进一步修饰, 并改用 刚性更强的醇进行醇解, 得到肉桂基酯 22, 经还原获得 中间体 $\mathbf{5}, e e$ 值 98\% (Scheme 7).

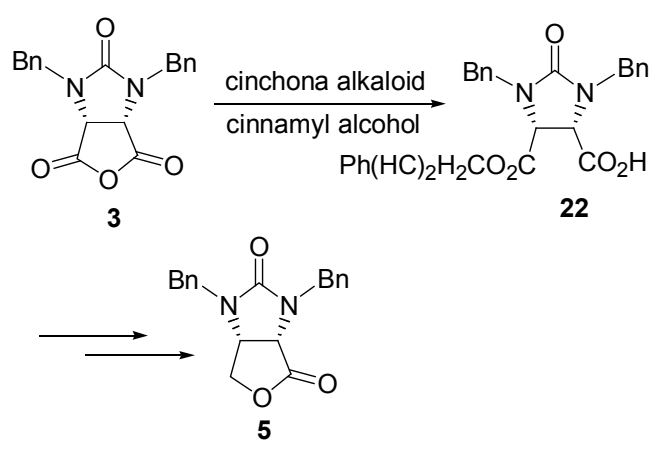

\section{Scheme 7}

之后 Chen 小组 ${ }^{[19]}$ 开发一种非常有价值的不对称催 化酯化的方法. 他们采用 (1S,2S)-1-(4-硝基苯基)-2- $N, N$ 二甲基氨基-3-三苯基甲氧基-1-丙醇作为催化剂(由氯霉
素中间体生产中产生的副产物转化而来), 在甲醇溶液 中不对称催化酯化酸䣶 3 得到半酯 4 , 再用嗍氢化钾-氯 化钙体系还原后, 酸化环合得到内酯 $\mathbf{5}$, 收率为 $88 \%$, ee 值达 98.5\%. 由于该方法是迄今以来获得内酯 $\mathbf{5}$ 最经济 简便的方法, 因此被应用于工业化生产，是目前 $(+)$ 生 物素工业生产的主要方法(Scheme 8).<smiles>O=C1OC(=O)[C@H]2[C@@H]1N(Cc1ccccc1)C(=O)N2Cc1ccccc1</smiles><smiles>COC(=O)[C@H]1[C@@H](C(=O)O)N(Cc2ccccc2)C(=O)N1Cc1ccccc1</smiles><smiles>O=C1OC[C@@H]2[C@H]1N(Cc1ccccc1)C(=O)N2Cc1ccccc1</smiles>

Scheme 8

此外, Chen 小组 ${ }^{[20]}$ 还尝试使用了生物催化合成中 间体 4 的方法. 酸 2 先酯化, 之后通过树脂固载的猪肝 水解酶得到 4, ee 值 $98 \%$ (Scheme 9).
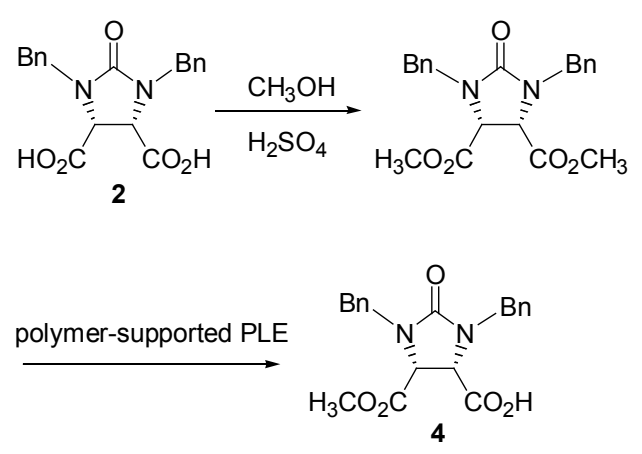

\section{Scheme 9}

除了上述以富马酸为起始原料合成关键中间体内 酯的路线外, 这些年来还有其他一些较有特色的方法被 报道.

2003 年 Schwarz 等 ${ }^{[21]}$ 公开了一条不对称合成路线. 他们首先将起始原料 5-羊基-2(5H)-呋喃酩(17)与(一)-薄 荷醇成醚得 18 , 然后与氯磺酸基异氭酸酯加成, 受薄荷 醇基手性诱导效应的影响, 立体选择性的得到 19, 在叠 氮化钠的亲核进攻作用下, 得到酰基叠氮化物 20, 在加 热条件下 20 发生重排得到嫝 21, 21 经过水解、还原和 上保护三步反应得到内酯 $\mathbf{5}$ (Scheme 10).

$(+)$-4-羊基噻吩并 $[3,4-d]$ 咪唑-2-酮 $(23)$ 是合成 $(+)$ 生物素的中间体之一, 可以通过氧化转化为内酯 $\mathbf{5}$. Huang 等 ${ }^{[22]}$ 报道了一种通过拆分将外消旋 $r a c-23$ 转化为 手性 23 的方法. 首先将 $r a c-23$ 通过邻苯二甲酸酐衍生 

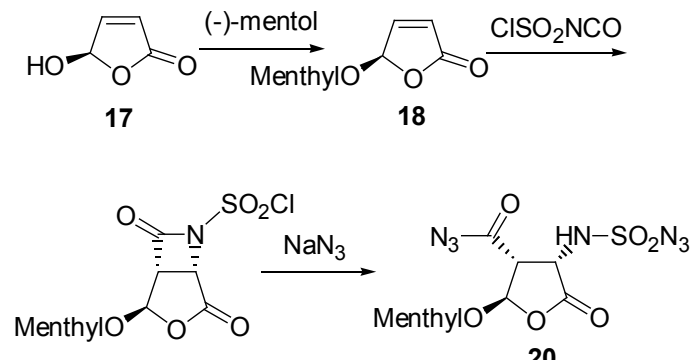

19

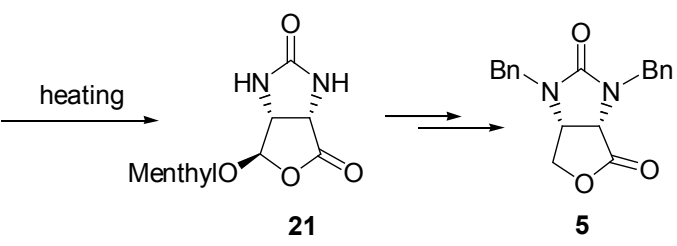

Scheme 10

化, 将得到的酸 rac-24 通过手性碱拆分剂进行拆分, 得 到 23, 重结晶后 $e e$ 值可达 98.5\% (Scheme 11).

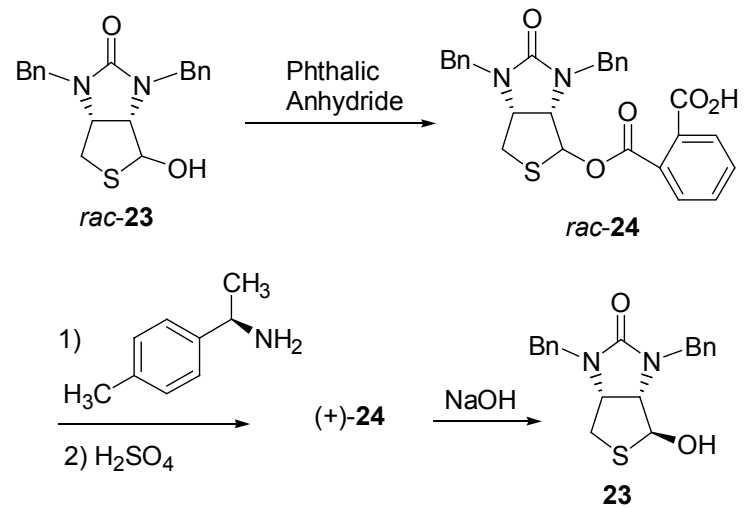

\section{Scheme 11}

将内酯 5 转化为硫内酯 $\mathbf{6}$ 的经典方法是用硫代乙酸 钾作硫代试剂, 收率达 $85 \%^{[5]}$. 然而工业化生产需要更 加经济的方法. 1995 年 Sumitomo 公司的研究人员发现 叔丁基黄原酸钾是很好的硫代试剂, 收率超过 90\%. 很 快他们 ${ }^{[23]}$ 又发现内酯 5 在乙酸钾、硫磺和硫酸和聚乙二 醇的存在下反应, 之后再在盐酸中用锌粉还原能同样高 收率的得到硫内酯 6. Chen 小组 ${ }^{[24]}$ 之后又报道了乙基黄 原酸钾，同期其他小组也报道了其他一些改进的硫代反 应体系，收率接近(Eq. 5).

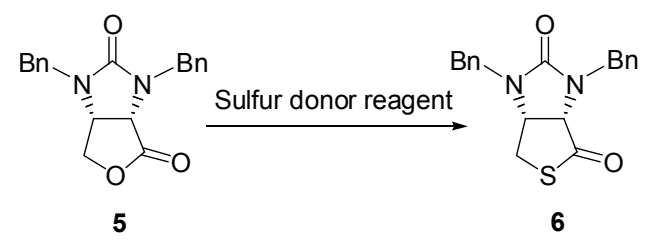

\section{2 羧基丁基侧链的引入}

由于格氏反应的实用性, 采用格氏反应引入翔
基丁基侧链是 $(+)$-生物素的工业化合成中的可靠方 法 ${ }^{[25]}$.

1999 年 Chen 小组 ${ }^{[26]}$ 报道了使用 3-乙氧基丙炔溴化 镁引入侧链的方法. 首先使用乙基溴化镁与 3-乙氧基丙 炔原位生成格氏试剂，与硫内酯偶联后得到 25 , 在溴化 镍一锌催化下选择性氢化还原之后重排为 26, 三步合计 收率 70\%, 26 可以像 7 一样转化为 $(+)$-生物素(Scheme 12).

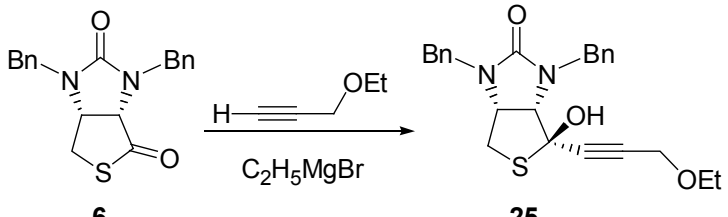

25

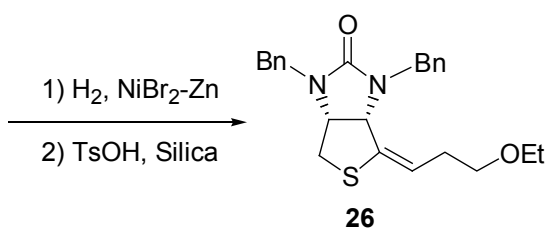

Scheme 12

2000 年 Chen 小组 ${ }^{[27}$ 还报道了另外一种通过格氏试 剂引入侧链的方法. 乙二醇保护的 5-溴-2-己酮制成格 氏试剂后与硫内酯 6 偶联后酸性条件下脱水得到 28 , 然 后通过碘仿反应得到 29, 两步合计收率 62\% (Scheme 13).

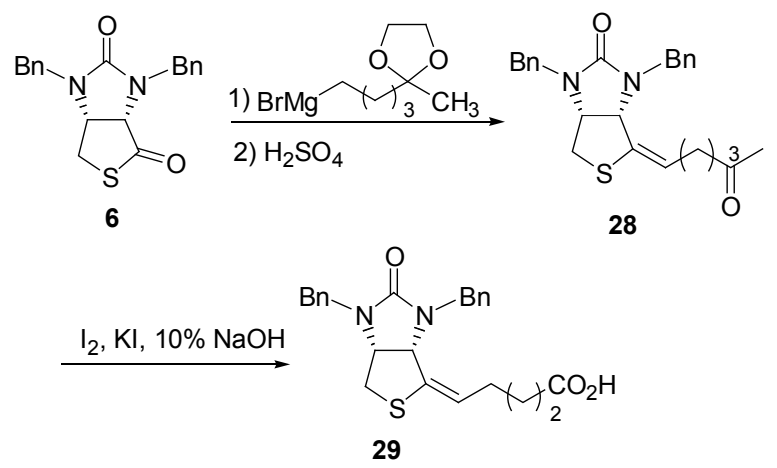

Scheme 13

Fukuyama 反应温和方便能专一性的得到 Z-构型产 物. Seki 小组 ${ }^{[28]}$ 将该反应用于 $(+)$-生物素的合成中. 他 们起初采用三甲基氯硅烷活化的锌试剂在二(三苯基膦) 氯化钯催化下与硫内酯 $\mathbf{6}$ 偶联得到中间体 30, 收率 $86 \%$. 由于钯配合物价格较高难于应用于工业生产，所 以经过改进该反应可在溴的活化下采用钯碳、乙酸钯或 者乙酰丙酮镍等多种催化剂催化与硫内酯偶联 ${ }^{[29]}$ (Eq. $6)$. 


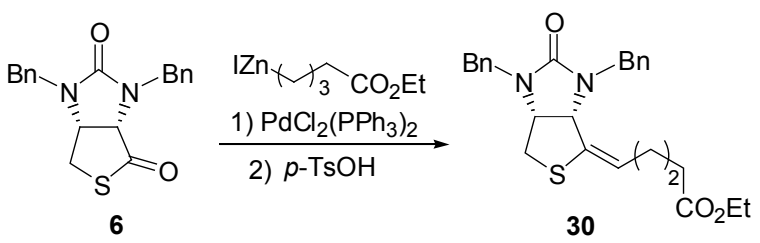

Wittig 反应是形成双键的经典方法. 2002 年 Chen 小 组 ${ }^{[30]}$ 报道, 用 DIBALH 先将硫内酯 6 立体选择性还原成 差基衍生物 23, 然后使用三苯基膦四氟硼酸氢盐季鏻 化得到相应的 Wittig 试剂 31, 与 5-氧代戊酸反应后得到 中间体 32, 三步合计收率 56\% (Eq. 7).<smiles>CCOC(=O)CC/C=C1\SC[C@@H]2[C@H]1N(Cc1ccccc1)C(=O)N2Cc1ccccc1</smiles>

Wittig 反应产生的副产物三苯氧磷难以处理, 为了 使反应后处理更适合大规模制备, Chen 小组 ${ }^{[20]}$ 之后报 道了利用 Horner-Emmons 反应引入侧链的方法. 硫内酯 6 首先通过与甲氧基甲基碘化镁反应后水解引入甲酰 基, 然后与二乙基 3-甲氧基羰基-2-丙烯基磷酸酯在氢 氧化钡存在下缩合得到酯 34, 34 在强碱作用下发生重排 并水解得到 35, 35 在氢氧化钯催化下氢化还原得 36 (Scheme 14).
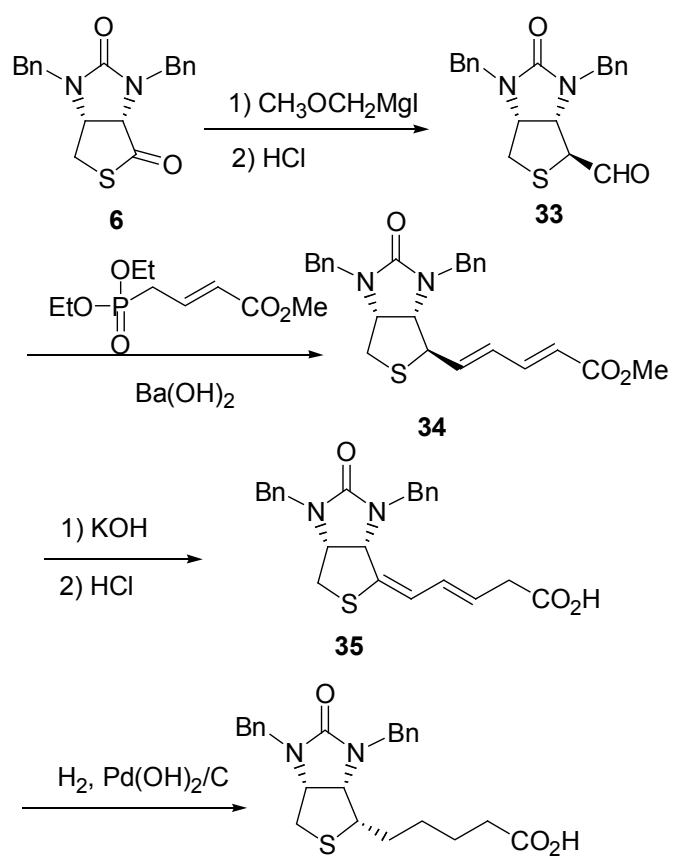

36

Scheme 14

由于大多数引入侧链的偶联反应得到是含双键的
中间体 29 或其类似物，为了更好的将双键选择性还原 成全顺式的产物, Sumitomo 公司的研究人员进行了一系 列研究. 1995 年他们发现乙酸钯可以作为催化氢化的催 化剂, 之后很快他们发现在反应中加入吸水的离子交换 络合树脂或多聚絮凝剂可以使反应操作更简便收率超 过 90\%. 2004 年有文献报道在合适 $\mathrm{pH}$ 值下使用钯碳催 化氢化, 反应效果更好 ${ }^{[31]}$ (Eq. 8).

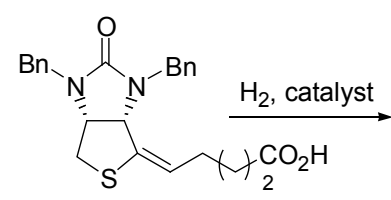

29

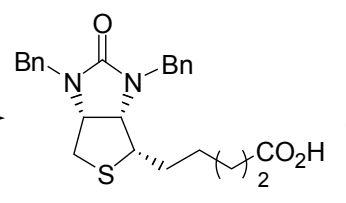

36

\section{3 双苄基 $(+)$-生物素的去保护基}

$(+)$-生物素合成中经典的方法是用氢溴酸溶液将 中间体双苠基生物素的苠基保护基脱去. 由于苠基与氮 原子相连的碳一氮键相当牢固，需要相当剧烈的条件才 能够断开. 在这种条件下 $(+)$-生物素的脲结构也会被相 当程度的破坏，因此反应产物往往较为复杂.

为了改善这一情况, Merck 公司的研究人员开发了 用浓硫酸脱去苠基保护基的方法. 之后 Sumitomo 公司 的研究人员将浓硫酸的浓度提高, 并使反应在两相中进 行，从而使反应更加温和，提高了产率 ${ }^{[32]}$ (Eq. 9).

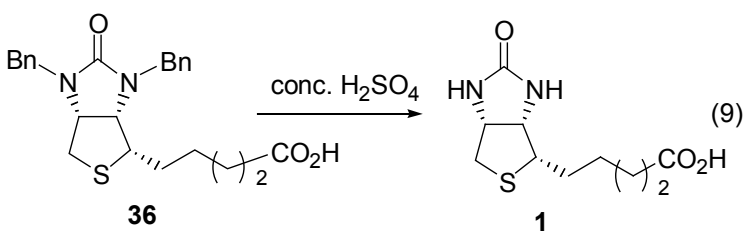

Chen 小组 ${ }^{[33]}$ 报道了使用次磷酸和碘的混合物能 较温和的将苄基脱去，产率中等.

之后 Chen 小组 ${ }^{[34]}$ 又发现，采用溴化氢反应体系时， 经过长时间完全反应后能得到单一的产物二胺溴化氢 盐 42, 42 经过与廉价的固体光气重新环合可以高收率的 得到 $(+)$-生物素，两步总收率高达 90\% (Scheme 15).

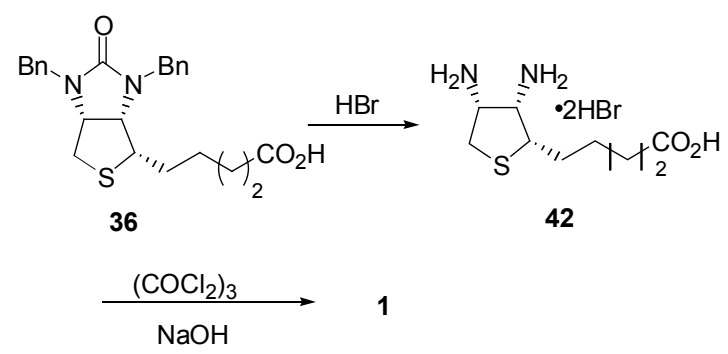

Scheme 15

\section{4 其他}

此外近年来一些与 HLR 类似的合成路线也得到了 
发展.

内酯 37 是内酯 5 的类似物, Brieden 等 ${ }^{[35]}$ 开发了一 条简便的合成路线. 首先将内酯 37 乙酰化然后硫代得 到硫内酯 39, 之后脱去乙酰基. 通过双格氏试剂偶联反 应再酸化水解的方法一次性引入侧链和末端羧基, 得到 (+)-生物素前体 41 (Scheme 16).
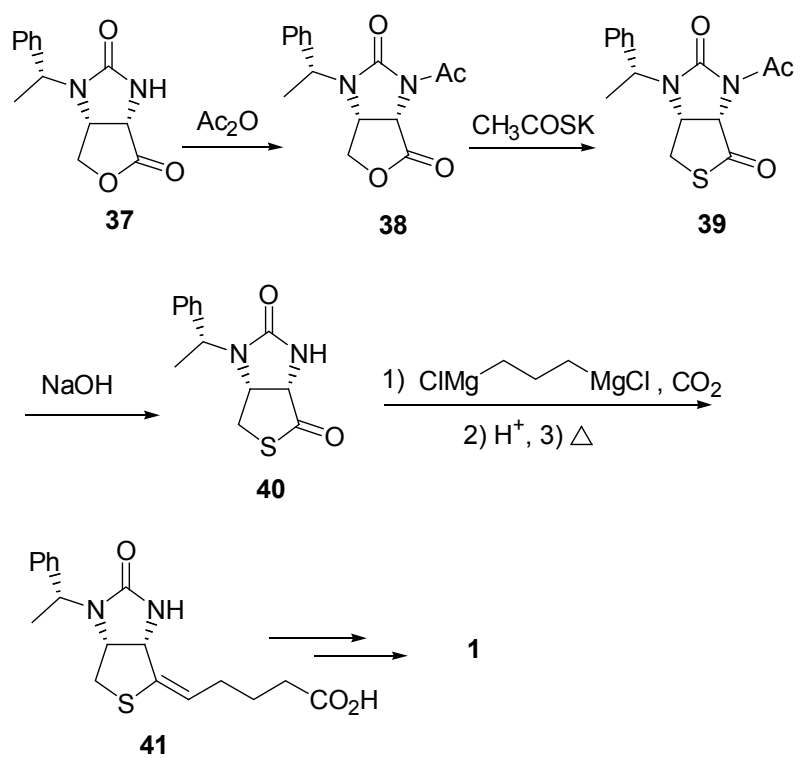

Scheme 16

\section{2 立体专一性合成策略(手性源策略)}

构建 $(+)$-生物素噻吩环上三个全顺式手性碳结构 需要借助手性试剂才能完成. 利用手性原料上的手性碳 原子, 通过结构转换得到 $(+)$-生物素, 则使得合成路线 更加简短巧妙.

\section{1 以 $L$-半胱氨酸/胱氨酸为起始原料的合成路线}

2001 年印度 Chavan 小组 ${ }^{[36]}$ 报道了一条(+)-生物素 的合成路线. $L$-胱氨酸首先转化为 $\mathbf{4 3}$, 被苄基异氧酸酯 酰基化后合环得到海因(hydantoin) 44, 使用 DIBAL-H 还原之后环合得到内半缩醛 45 , 然后在酸性条件下开 环, 羟基被苯硫酚取代得醛 46, 之后用强碱 DBU 烯醇 化后被三甲基硅基保护得烯醇硅醚 47. 最后在路易斯 酸三甲基硅基三氟甲磺酸酯催化下与对硝基苯甲醛反 应, 苯硫基离去而噻吩环关环得到醛 33. 醛 33 可以通 过 Wittig 反应或 Horner-Emmons 反应引入羧基丁基侧链 得到(+)-生物素 (Scheme 17).

2002 年 Seki 小组 ${ }^{[37]}$ 报道了一条以立体选择性氧硅 基化和钯催化分子内烯丙基氨基化为关键步骤的全合 成路线. $L$-半胱氨酸首先通过四步反应转化为 49 , 然后 在三丁基膦催化下与氧基三甲基硅烷发生氰硅基化反 应立体选择性的得到硅醚 50. 然后通过双格氏试剂引 入羧基丁基侧链, 经过水解脱掉硅保护基再酯化得酯
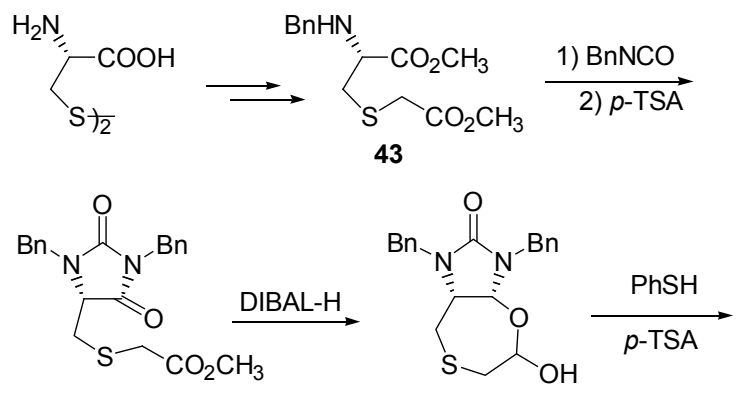

44

45

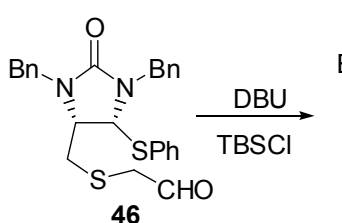

46

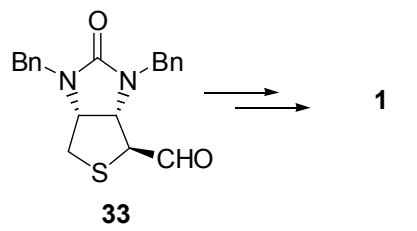

Scheme 17

51. 经酰基化保护差基后, 51 上四氢噻唑环在酸性条件 下开环，然后利用还原氨化将游离出的氨基用芐基保 护，而游离出的颈基与羰基缩合后经过脱水反应得到酯 53.53 在乙酸钯催化下发生烯丙基氨化反应得到生物素 前体 54.54 经过简单几步反应可以得到目标产物，总收 率 25\% (Scheme 18).

2003 年 Seki 小组 ${ }^{[38]}$ 又报道了一条合成关键中间体 硫内酯 $\mathbf{6}$ 的路线. $L$-半胱氨酸首先经三步反应得到 $(R)-N-$ 芐基-2-噻唑酮-4-醛 $\mathbf{5 5}$, 醛 55 与苠基胺和三甲基氧硅烷 发生 Strecker 反应高立体选择性的得到 $\alpha$-氨基氰 56 (syn：anti=28：1). 氨基氰 56 经 Katritzky 法水解得酰 胺 57. 后者在加热情况下发生羰基转移, 之后酸性水解 得颈基羒酸 58. 用 DCC 作缩合剂 $\mathbf{5 8}$ 环合后生成硫内酯 6, 合计收率 49\% (Scheme 19).

同时 Seki 小组 ${ }^{[39]}$ 又报道了另一条殊途同归的路线. $L$-半胱氨酸首先转化成 $\mathbf{5 9}, \mathbf{5 9}$ 采用苯氧羰基保护后使用 三氧化硫-吡啶复合物氧化得到醛 61, 61 同样经过 Strecker 反应和 Katritzky 法水解得到酰胺 63. 在加热条 件下 63 发生分子内酰基化反应得到酰胺 64, 64 在乙酸 中被锌还原，芐位碳硫键断裂，之后水解的颈基羧酸 58, 合计收率 21\% (Scheme 20).

2005 年 Chavan 小组 ${ }^{[40]}$ 又报道了一条新路线. 首先 按照 Poetsh 法由 $L$-半胱氨酸制备了双环海因 65 , 海因 65 被硼氢化钠还原得羟基咪唑并噻吩酮 66, 在三氟化 嗍-乙醚的催化下与 1,2-双 (三甲基硅氧基)环已烯缩合 


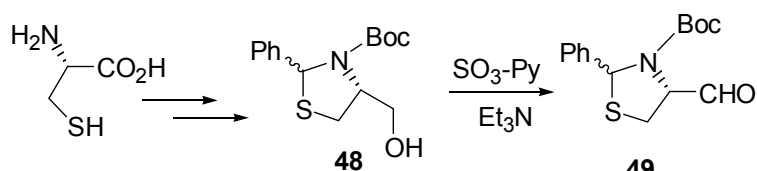

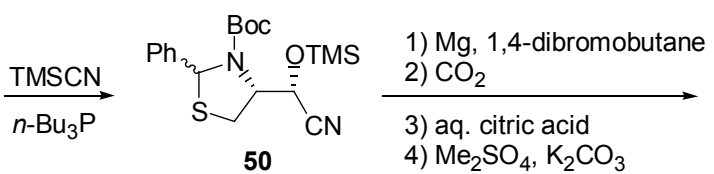

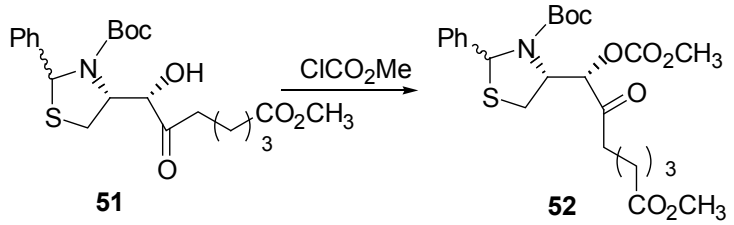

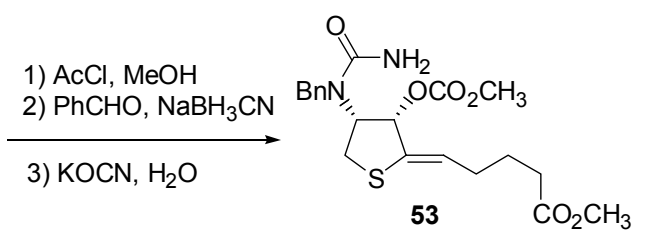

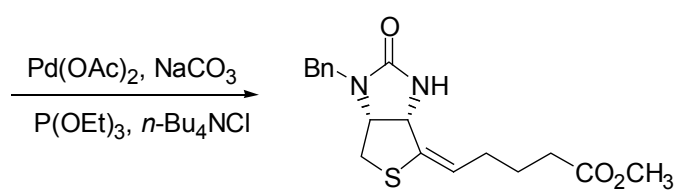

54

\section{Scheme 18}

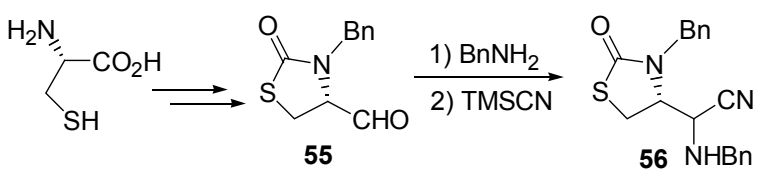

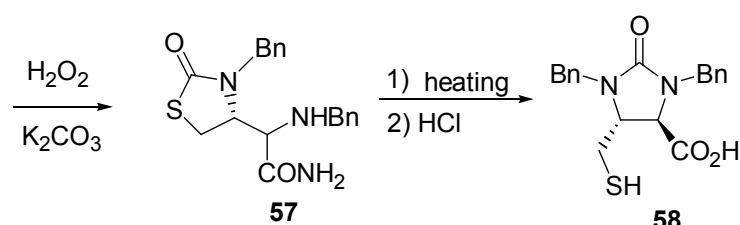<smiles>O=C1SC[C@@H]2[C@H]1N(Cc1ccccc1)C(=O)N2Cc1ccccc1</smiles>

Scheme 19

得到 $\alpha$-着基酮 67. 一般认为高度的立体选择性是由于 咪唑并噻吩酩平面的两边位阻不同造成的. 使用叔丁基 过氧化氢将 67 通过 Bayer-Villiger 反应氧化, 之后酯化 得到酮酯 68. 68 在乙酸中被锌粉还原游离出䘪基, 放基 与酮缩合脱水后得到 $(+)$-生物素前体 69, 最后以 $20 \%$ 的总收率得到 $(+)$-生物素(Scheme 21).

Takahashi 等 ${ }^{[41]}$ 在 2001 年曾经报道过一条简短的路

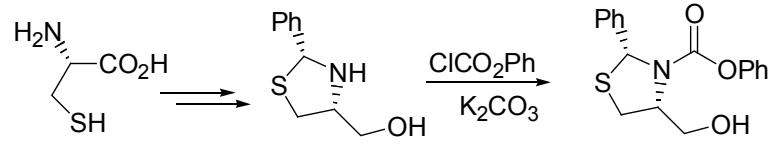

60

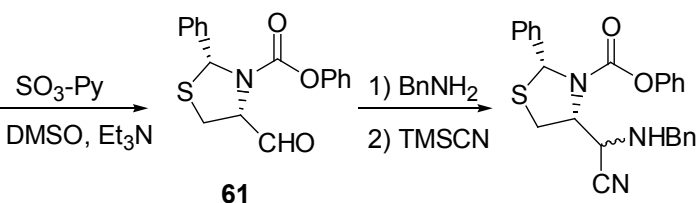

62

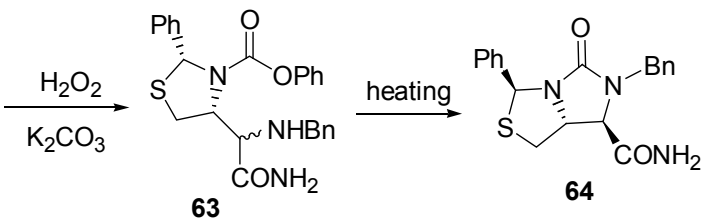<smiles>O=C(O)C(C(=O)O)C(=O)O</smiles>

Scheme 20<smiles>CC(C)[13CH2]Cc1ccccc1</smiles>

1,2-bis(trimethylsilyoxy)cyclohexene<smiles>O=C1N(Cc2ccccc2)[C@H](C2(O)CCCCC2=O)[C@@H]2CS[C@@H](c3ccccc3)N12</smiles>

67 $\underset{\text { 1) } \mathrm{TBHP}, \mathrm{KOH}-\mathrm{MeOH}}{\stackrel{\mathrm{CH}_{2} \mathrm{~N}_{2}}{\longrightarrow}}$

$\underset{\text { 1) } \mathrm{Zn} / \mathrm{AcOH}}{\text { 2) } \mathrm{AcOH} / \text { piperidine }}$
69

Scheme 21

线，该路线之后被改进的更加实用化. 首先按照 Poetsh 法合成出海因 65 , 然后在乙酸中用锌粉还原得放基酰 胺 70, 70 用已二酸单甲酯酰氯酰基化得 71, 然后在 $\mathrm{Zn}$ 粉- $\mathrm{TiCl}_{4}$ 催化下缩合得到 $\mathbf{7 2}$, 最后经催化氢化和脱苠基 以 29\%收率得到(+)-生物素(Scheme 22). 
<smiles>N[C@@H](CS)C(=O)OCC#CC(=O)O</smiles><smiles>CC(=O)OCCC(=O)SCC1C(=O)N(Cc2ccccc2)C(=O)N1Cc1ccccc1</smiles><smiles>CC(=O)CCCCC1=C2C(C)SCC2N(Cc2ccccc2)C(=O)N1Cc1ccccc1</smiles>

72

Scheme 22

\section{2 以 $D-(+)$-氨基葡糖和 $L-$-天门冬氨酸为起始原料的} 合成路线

2004 年 Chavan 小组 ${ }^{[42]}$ 报道了一条以氨基葡萄糖为 起始原料的路线. $D-(+)$-氨基葡萄糖首先被异氯酸苠酯 酰基化, 然后在吡啶催化下发生糖苷化反应得到 cis-呋 喃型糖苷 73. 用异丙亚基将 73 上羟基保护后，再用茮 基将酰胺保护得 74. 然后在酸性条件下将异丙亚基去 保护, 用高碘酸将邻二醇氧化断裂得到相应醛后用在碱 性条件下烯醇化并用乙酸酐酰化得乙酸烯醇酯 76. 在 $\mathrm{BF}_{3}-\mathrm{Et}_{2} \mathrm{O}$ 催化下与三甲基氰硅烷反应得腈 77 , 用嗍氢 化钠将 77 还原并在三甲基氯硅烷-甲醇体系中脱乙酰基 将腈转化为酯 $\mathbf{7 8}$, 再次使用高碘酸氧化断裂得到醛并 用乙二醇保护得缩醛 79, 钯碳催化氢解脱苠基后将酯 还原成醇 81,81 被转化为磺酸酯后, 被硫化钠取代然后 再水解脱去乙二醇保护最后得到醛 33. 醛 33 可以通过 已报道方法转化为 $(+)$-生物素，总收率 $25 \%$ (Scheme 23).

$L$-天门冬氨酸是首次被用于合成 $(+)$-生物素. $L$-天 门冬氨酸首先转化成苠氧羰基保护的内酯 82 , 通过羟 醛缩合在 $\alpha$-位引入羟甲基并用茮基氧甲基保护得到内 酯 83, 用氨水开环得酰胺 84, 用 Jones 试剂将差基氧化 成着基后通过 Hofmann 重排将酰胺转化为氨基, 然后分 子内酰基化关环得到 $\mathbf{8 5}$, 用氢氧化钯将苠基脱去后关 环得到内酯 22. 22 可以被茮基保护得到关键中间体内酯 5, 总收率 $12 \%{ }^{[43]}$ (Scheme 24).

\section{3 结论与展望}

$(+)$-生物素在市场中的重要地位需要更加高效的 合成路线和经济的原料. 不对称合成策略的发展是这十 余年来的改进研究工作最大的特色. 就 HLR 路线而言,
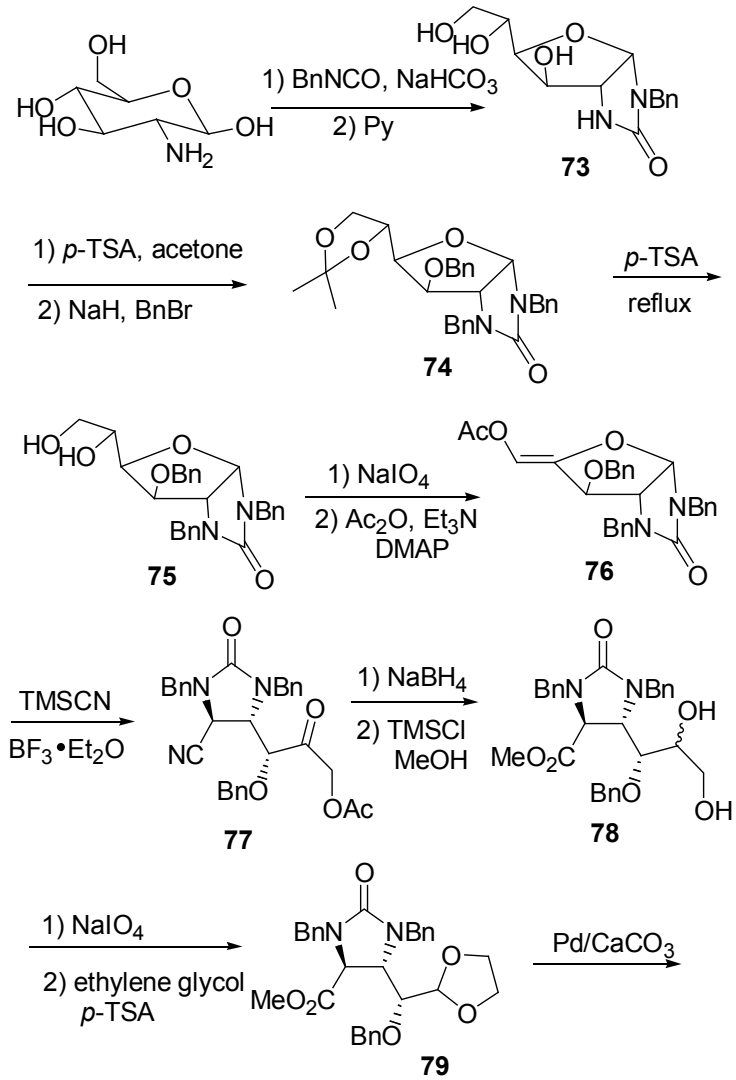

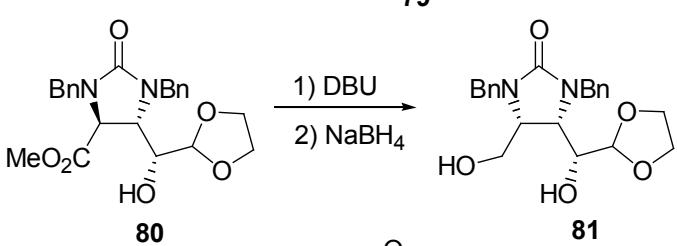

1) $\mathrm{MsCl}, \mathrm{Et}_{3} \mathrm{~N}$, DMAP

2) $\mathrm{Na}_{2} \mathrm{~S}$

3) $\mathrm{HCl}, \mathrm{CH}_{3} \mathrm{CO}_{2} \mathrm{H}$<smiles>O=C[C@@H]1SC[C@H]2[C@@H]1N(Cc1ccccc1)C(=O)N2Cc1ccccc1</smiles>

Scheme 23

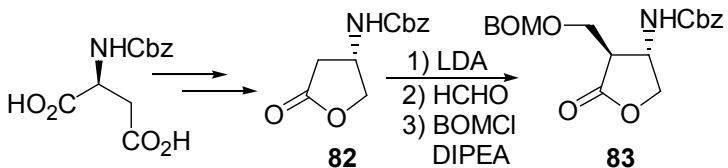

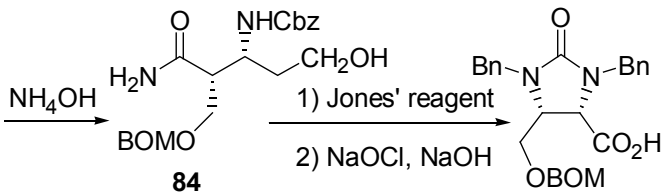

85<smiles>O=C1OC[C@@H]2NCN[C@@H]12</smiles> 
主要研究集中在如何建立三个连续的手性碳上. 关键中 间体内酯 $\mathbf{5}$ 不对称催化合成的巨大成功使得 $(+)$-生物素 的生产成本显著降低, 是该领域内最为重要的进展. 但 是挑战(+)-生物素全合成的工作远没有结束. 例如直接 将酸酐 3 不对称还原为内酯 5 等设想仍然有待证实. 同 时 $L$-半胱氨酸被认为是手性源策略最理想的起始原料, 手性原料本身具有的优势结构因素能够很大程度的规 避不对称合成策略中的难点. 实际上该策略发展的已经 非常实用化，有希望未来挑战 HLR 路线在工业化生产 中的地位.

\section{References}

[1] Uskokovic. M. R. Biotin in Encyclopedia of Chemical Technology, Vol. 24, Eds.: Kirk, R. E.; Othmer, D. E., Wiley, New York, 1984, p. 41.

[2] (a) Minko, T.; Paranjpe, P. V.; Qiu, B.; Lalloo, A.; Won, R.; Stein, S.; Sinko, P. J. Cancer Chemother. Pharmacol. 2002, 50, 143.

(b) Green, N. M. Biochem. J. 1963, 89, 585.

(c) Melamed, M. D.; Green, N. M. Biochem. J. 1963, 89, 591

(d) Green, N. M. Adv. Protein Chem. 1975, $29,85$.

(e) Fuhr, J. P. Jr.; He, H.; Goldfarb, N.; Nash, D. B. Dis. Manage. 2005, 8, 265.

[3] De Clercq, P. J. Chem. Rev. 1997, 97, 1755.

[4] (a) Goldberg, M. W.; Sternbach, L. H. US 2489232, 1951 [Chem. Abstr. 1951, 45, 184].

(b) Goldberg, M. W.; Sternbach, L. H. US 24892351951 [Chem. Abstr. 1951, 45, 186a].

(c) Goldberg, M. W.; Sternbach, L. H. US 24892381951 [Chem. Abstr. 1951, 45, 186g].

[5] (a) Chen, F.-E.; Ling, X.-H.; Lu, Y.-X.; Zhang, X.-Y.; Peng, X.-H. Chem. J. Chin. Univ. 2001, 22, 1141 (in Chinese).

(陈芬儿, 凌秀红, 吕银祥, 张晓跃, 彭晓华, 高等化学学报, 2001, 22, 1141.)

(b) Chen, F.-E. CN 1443766, 2003 [Chem. Abstr. 2003, 143, 326351].

[6] Zhang, G.-J.; Chen, J.-H.; Wu, Z.-G.; Zhang, F.; Shen, R.-P. Chin. J. Pharm. 2009, 40, 168 (in Chinese).

(张国钧, 陈建辉, 吴志刚, 张符, 沈润溥, 中国医药工业杂志, 2009, 40, 168.)

[7] Matsuki, K.; Inoue, H.; Takeda, M. Tetrahedron Lett. 1993, 34, 1167.

[8] Chen, F.-E.; Huang, Y.-D.; Fu, H.; Cheng, Y.; Zhang, D.-M.; Li, Y.-Y.; Peng, Z.-Z. Synthesis 2000, 2004.

[9] Ostendorf, M.; Romagnoli, R.; Pereiro, J. C.; Roos, E. C.; Moolenaar, M. J.; Speckamp, W. N. Tetrahedron: Asymmetry 1997, 11, 1773.

[10] (a) Shimizu, M.; Nishigaki, Y.; Wakabayashi, A. Tetrahedron Lett. 1999, 40, 8873.

(b) Tamotsu, F.; Shimizu, M. JP 2000256360, 2000 [Chem. Abstr. 2000, 133, 237771].

[11] Chen, F.-E.; Dai, H.-F.; Kuang, Y.-Y.; Jia, H. Q. Tetrahedron: Asymmetry 2003, 14, 3667.

[12] Chen, F.-E.; Yuan, J.-L.; Dai, H.-F.; Kuang, Y.-Y.; Chu, Y. Synthesis 2003, 2155.

[13] Chen, F.-E.; Jia, H.-Q.; Chen, X.-X.; Dai, H.-F.; Xie, B.; Kuang, Y.-Y.; Zhao, J.-F. Chem. Pharm. Bull. 2005, 53, 743.

[14] Wehrli, C. WO 2004094367, 2004 [Chem. Abstr. 2004, 141, 395554].
[15] Schwarz, M.; Eckstein, J. WO 2001025215, 2001 [Chem. Abstr. 2001, 134, 281013].

[16] (a) Choi, C.; Tian, T.-S.; Deng, L. Synthesis 2001, 1737. (b) Deng, L.; Liu, X.-F. WO 2004110609, 2004 [Chem. Abstr. 2004, 141, 363443].

[17] Florni, M.; Porcheddu, A.; Taddei, M. Tetrahedron Lett. 1999, 40, 4395.

[18] (a) Xiong, F.; Chen, X.-X.; Chen, F.-E. Tetrahedron: Asymmetry 2010, 21, 665 .

(b) Xiong, F.; Chen, X.-X.; Liu, Z.-Q.; Chen, F.-E. Tetrahedron Lett. 2010, 51, 3670.

(c) Dai, H.-F.; Chen, X.-X.; Zhao, L.; Sheng, H.; Chen, F.-E. Adv. Synth. Catal. 2008, 350, 1635.

[19] Chen, F.-E. CN 1473832, 2004 [Chem. Abstr. 2004, 142, 373827].

[20] Chen, F.-E.; Jia, H.-Q.; Chen, X.-X.; Dai, H.-F.; Xie, B.; Kuang, Y.-Y.; Zhao, J.-F. Adv. Synth. Catal. 2005, 347, 549.

[21] Schwarz, M.; Heywang, U.; Kuppe, T. WO 2002004460, 2002 [Chem. Abstr. 2002, 136, 102234].

[22] Lu, Y.-X.; Huang, Y.-M.; Huang, Y.-F.; Lu, Y. Synth. Commun. 2002, 32, 781.

[23] (a) Yoshikawa, K.; Kurimoto, I.; Sakito, Y. JP 08217779, 1996 [Chem. Abstr. 1996, 125, 328709].

(b) Hirata, N.; Miyamoto, Y.; Takahashi, T. JP 10231298, 1998 [Chem. Abstr. 1998, 129, 216619].

(c) Yani, N.; Seko, S. JP 09263487, 1997 [Chem. Abstr. 1997, 127, 476676].

(d) Uemura, T.; Shirahta, M.; Miyamoto, Y. JP 11101235, 1999 [Chem. Abstr. 1999, 131, 264751].

[24] (a) Chen, F.-E.; Peng, X.-H.; Sun, G.-F. CN 1358725, 2002 [Chem. Abstr. 2002, 139, 381479].

(b) Lu, X.-Y. CN 1548440, 2004 [Chem. Abstr. 2004, 142, 772178].

[25] Koop, U.; Krummradt, H.; Schwarz, M.; Stoldt, J.; Eckstein, J.; Zehner, S.; Melichnar, W. DE 10001317, 2001 [Chem. Abstr. 2001, 135, 107188].

[26] Chen, F.-E.; Peng, Z.-Z.; Shao, L.-Y.; Cheng, Y. Acta Pharm. Sin. 1999, 34, 822 (in Chinese).

(陈芬儿，彭作中，邵兰英，程爆，药学学报, 1999, 34, 822.)

[27] Chen, F.-E.; Huang, Y.-D.; Fu, H.; Cheng, Y.; Zhang, D.-M.; Li, Y.-Y.; Peng, Z.-Z. Synthesis 2000, 2004.

[28] (a) Seki, M.; Shimizu, T. JP 2000191665, 2000 [Chem. Abstr. 2000, 133, 73893m].

(b) Seki, M.; Shimizu, T. Tetrahedron Lett. 2000, 41, 5099.

[29] (a) Kimura, M.; Seki, M. Tetrahedron Lett. 2005, 45, 1635; (b) Mor, Y.; Seki, M. Synlett 2005, 2233.

[30] Chen, F.-E.; Fu, H.; Meng, G.; Luo, Y. F.; Yan, M. G. Chem. J. Chin. Univ. 2002, 23, 1060 (in Chinese). (陈芬儿，傅晗，孟歌，罗有富，漂明国，高等学校化学学报， 2002, 23, 1060.)

[31] (a) Takahshi, T.; Myamoto, Y.; Mizuno, M.; Hirata, N.; Mizuno, T. JP 07330776, 1995 [Chem. Abstr. 1995, 124, 875668].

(b) Takahshi, T.; Myamoto, Y.; Mizuno, M.; Hirata, N. US 5859260, 1996 [Chem. Abstr. 1996, 126, 576441].

(c) Lehmann, S.; Koppe, T. US 20040030150, 2004 [Chem. Abstr. 2004, 142, 266823].

[32] (a) Heywang, U.; Bollinger, H.; Müller, H. R. WO 9843979, 1998 [Chem. Abstr. 1998, 129, 275786].

(b) Kiyoshima, Y.; Miyamoto, Y. JP 10367060, 1998 [Chem. Abstr. 1998, 128, 441734].

(c) Lin, D.; Liu, D.; Lu, X. CN 1778803, 2006 [Chem. Abstr. 2006, 146, 358894].

[33] Meng, G.; He, Y.-P.; Chen, F.-E. Synth. Commun. 2003, 33, 2593. 
[34] (a) Chen, F.-E. CN 1374312, 2002 [Chem. Abstr. 2002, 140, 181254].

(b) Deshpade, P. B.; Holkar, A. G.; Karale, S. N.; Jafri, W. S. WO 2004041430, 2004 [Chem. Abstr. 2004, 142, 773110].

[35] Brieden, W.; McGarrity, J. WO 9815557, 1998 [Chem. Abstr. 1998, 128, 308348].

[36] (a) Chavan, S. P.; Tejwani, R. B.; Ravindranathan, T. J. Org. Chem. 2001, 66, 6197.

(b) Ravindranathan, H.; Chavan, S. B.; Subhash, P.; Rajkumar, R. B. IN 178927, 1998 [Chem. Abstr. 1998, 140, 303667].

[37] (a) Seki, M.; Hatsuda, M.; Mori, Y.; Yamada, S. Tetrahedron Lett. 2002, 43, 3267.

(b) Seki, M.; Hatsuda, M.; Mori, Y.; Yamada, S. J. Org. Chem. 2002, 67, 5527.

[38] (a) Seki, M.; Kimura, M.; Hatsuda, M.; Yashida, S.; Shimizu, T. Tetrahedron Lett. 2003, 44, 8905.

(b) Seki, M.; Kimura, M.; Hatsuda, M.; Yashida, S.; Yamada, S.; Shimizu, T. Chem. Eur. J. 2004, 10, 6102.

(c) Seki, M.; Hatsuda, M.; Yashida, S.; Shimizu, T. Tetrahedron Lett. 2004, 45, 6579.

(d) Seki, M.; Kimura, M.; Hatsuda, M.; Yashida, S.; Shimizu, T.
US 20050038260, 2005 [Chem. Abstr. 2005, 144, 465455].

(e) Seki, M. WO 2004089878, 2004 [Chem. Abstr. 2004, 141, 365968].

[39] Mori, Y.; Kimura, M.; Seki, M. Synthesis 2003, 2311.

[40] (a) Chavan, S. P.; Chittiboyina, A. G.; Ravindranathan, T.; Kamat, S. K.; Kalkote, U. R. J. Org. Chem. 2005, 70, 1901.

(b) Chavan, S. P.; Chittiboyina, A. G.; Amar, G.; Kamat, S. K.; Kalkote, U. R.; Ravindranathan, T. EP 1219625, 2002 [Chem. Abstr. 2002, 137, 63120].

(c) Chen, K.-X.; Cheng, X.-W.; Jin, J.-M.; Sheng, L.-Q. Chin. J. Org. Chem. 2006, 26, 1309 (in Chinese).

(陈克喜, 程向炜, 金佳敏, 盛柳青, 有机化学, 2006, 26, 1309.)

[41] (a) Takahashi, M.; Sakurai, O.; Kokubo, O. S. H. JP 2001002679, 2001 [Chem. Abstr. 2001, 134, 100868].

(b) Zhao, J.; Li, Y. B.-Q.; Zhang, S. Fine Chem. 2004, 21, 30 (in Chinese).

(赵嘉, 李云政, 张青山, 精细化工, 2004, 21, 30.)

[42] Chavan, S. P.; Ramakrishna, G.; Gonnade, R. G.; Bhadbhade, M. M. Tetrahedron Lett. 2004, 45, 7307.

[43] Seki, M.; Shimizu, T.; Inubushi, K. Synthesis 2002, 361. 